\title{
Wissen sammeln, Wissen teilen
}

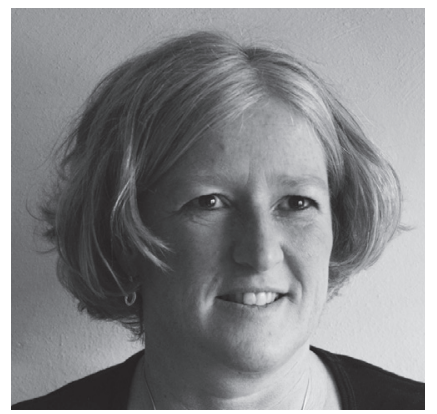

VON SILKE DEGENHARDT Silke Degenhardt verfügt über 20 Jahre Erfahrung in unterschiedlichen Bereichen der Sozialwirtschaft, davon seit zwölf Jahren in der Informationstechnologie. Nach beruflichen Erfahrungen in der Beratung und Schulung bei Softwareunternehmen verantwortet sie bei einem mittelständischen Unternehmen der Sozialwirtschaft als kommissarische Leiterin die IT. Die Diplom-Sozialarbeiterin ist Absolventin des ersten Masterstudiengangs Sozialinformatik an der Katholischen Universität Eichstätt-Ingolstadt. E-Mail silke.degenhardt@gmail. com

\author{
Über Wissensmanagement wurde in den \\ letzten Jahrzehnten viel geredet - und wenig \\ getan. Zunächst sollte es die Technik richten, \\ dann die Organisationsentwickler. Doch die \\ Informationstechnologie (IT) war lange Zeit zu spröde \\ und die Berater für viele Sozialträger zu teuer. Heute \\ lautet die Frage: Was taugen die neuen Impulse aus \\ der Mitmach-Kultur des Internets? Und wie kann \\ man sie für Unternehmen nutzbar machen?
}

Seit den 1990er Jahren gibt es viele Bestrebungen, den Umgang mit Wissen im Unternehmen besser zu organisieren. Diese waren zunächst stark technologisch geprägt. Die Innovationen im ITBereich und Vernetzungsmöglichkeiten durch das Internet ermöglichten dies. Viele Projekte scheiterten jedoch deshalb, weil die eigentlichen Wissensträger, die Mitarbeiterinnen und Mitarbeiter, schlicht vergessen wurden. Die heutige Sicht auf das Wissensmanagement men müssen. Das Wissen der sozialen Organisationen ist in weiten Bereichen kaum visualisierbar und darstellbar es handelt sich um implizites Wissen. Es ist stark an die beteiligten Personen und deren individuellen Erfahrungen gekoppelt.

Explizites Wissen hingegen kann über die unterschiedlichsten Medien dargestellt werden, es ist »sichtbar", was jedoch nicht gleichbedeutend damit ist, dass es auch genutzt wird. Beispiele sind

\section{"Die Unternehmenskultur ist der zentrale Erfolgsfaktor für Wissensmanagement»}

ist umfassender. Die Technik steht nun weniger im Vordergrund, ist jedoch für Teilprozesse unverzichtbar.

Soziale Unternehmen stehen heute als Dienstleister für Menschen vor ähnlichen Herausforderungen wie die gewerbliche Wirtschaft. Fachkräftemangel, zunehmender Wettbewerbsdruck und die Beschleunigung durch die geringere »Halbwertzeit « von Wissen und Informationen sorgen dafür, dass Unternehmen sich dieser Themen anneh-
Fachliteratur, Dokumentationen, Handbücher zum Qualitätsmanagement. Der Weg, implizites Wissen darstellbar zu machen und zu explizieren, verläuft über Kommunikation, Interaktion und Zusammenarbeit zwischen den Beteiligten.

Die Web 2.0-Technologien wie Wikis, Blogs, Foren, Social Media leben von der Kommunikation und Interaktion, Menschen beteiligen sich unterschiedlich aktiv an Themen. Hierzu einige 


\section{Fünf Beispiele für Wissensmanagement}

Drei Instrumente mögen als Beispiele für mögliche Anwendungen im Wissensmanagement dienen:

- "Yellow Pages" sind eine Art Gelbe Seiten (Telefonbuch) und zeigen das Wissen von Mitarbeitenden auf, sie legen verborgenes Wissen offen. Ziel ist das Erstellen von Kompetenzprofilen des personenbezogenen Wissens.

- "Communities of practice" sind lose Formen von Gruppenarbeit, die sich mit einem bestimmten Thema befassen. Sie arbeiten eigenverantwortlich und steuern sich selbst. Ihr Ziel ist Wissen auszutauschen, zu verteilen und durch die Zusammenarbeit neues Wissen zu entwickeln.

- "Lessons Learned " lassen sich als Baustein in das Projektmanagement einbauen. Es kann als festes Element zur Evaluation in die Ergebnissicherung (oder Zwischenbewertung) von Projekten eingebaut werden. Dabei geht es darum negative, aber auch positive Aspekte bei der Bearbeitung des Projektes nachvollziehbar zu beschreiben, so dass andere daraus lernen können.

Zwei zusätzliche Beispiele können zeigen, wie speziell Web 2.0-Technologien im Wissensmanagement genutzt werden können:

- Projektarbeit: Im Projekt nutzen die Teilnehmenden eine Netzwerkplattform, in der alle Daten abgelegt werden. Sie sind für alle Teilnehmerinnen und Teilnehmer das führende Werkzeug zur Organisation der Zusammenarbeit. Projektauftrag, Zeitpläne, Dokumentationen, Protokolle, Links und Adressen werden dort gespeichert. Termine und Aufgaben sowie die Statusverfolgung der Aufgaben werden im Netz hinterlegt und ständig aktualisiert. Über Änderungen werden die Nutzer automatisch per E-Mail verständigt. Im Forum werden Ideen gesammelt und diskutiert, welche in der nächsten Projektsitzung zeitsparend bearbeitet werden. Auf einer Wikiseite werden die Ergebnisse des Projektes von den Teilnehmenden beschrieben mit Bild, Text und Ton. Die Projekt-Wikiseite wird nach Abschluss des Projektes unternehmensintern veröffentlicht. Im Projekt werden positive und negative Aspekte reflektiert und auf der zentralen Projektseite veröffentlicht.

- Fortbildungen: Fortbildungen werden in einem Portal angeboten und gebucht. Sie sind mit Metadaten kategorisiert. Die Auswertung der Fortbildung erfolgt im Portal über eine Teilnehmerbefragung (Umfrage). Dokumentationen und Präsentationen werden zum Nachlesen eingestellt. Von jeder abgeschlossenen Fortbildung stellen Teilnehmerinnen und Teilnehmer einen kurzen Bericht ins Netz, der sich an einer vorgegebenen Struktur orientiert (Erwartung: Was habe ich erwartet. Lerneffekt: Was habe ich daraus gelernt. Verbesserung: Was könnte im Team, im Arbeitsbereich verbessert werden). Die Darstellung der Kurzberichte erfolgt über eine Tabelle, in der die Inhalte mit Metadaten verschlagwortet werden, damit sie später in der Suche einfach aufgefunden werden können. Die Verbesserungsvorschläge werden zentral analysiert und bewertet und es gibt Rückmeldungen an die Benutzer.

Silke Degenhardt werden kann. Dokumenten-Managementsysteme, Portal- und GroupwareLösungen sowie Datenbanken bieten verschiedene Lösungsansätze an. Produkte wie der Microsoft SharePoint Server fassen verschiedene Funktionalitäten zusammen. Unterschiedliche Suchfunktionen ermöglichen das Wiederauffinden von Informationen.

Bei aller kommunikativen Stärke der Web 2.0-Funktionen liegt hier ihr entscheidender Schwachpunkt. Informationen liegen verstreut im Netz, eine strukturierte Darstellung der Inhalte ist kaum möglich. Das Wissen und die Informationen, die auf diese Weise ausgetauscht werden, in kontrollierte Bahnen zu lenken, steuern, komprimieren und zusammenzufassen, gleicht einer Quadratur des Kreises. Im Sinne eines Managements von Information und Wissen ist dies jedoch unerlässlich.

Neben den IT-Systemen sind jedoch die kulturellen Bedingungen in der Organisation und die Rolle des Managements wesentlich entscheidender. Kommunikationskultur und Informationspolitik, die Führungsgrundsätze und Veränderungswege im Unternehmen sind wichtige Faktoren. Starre Hierarchien sind in diesen Prozessen eher hinderlich. Förderlich ist hingegen eine Projektkultur. Hier gibt es viele Ansätze und Instrumente des Wissensmanagements, die sich gut IT-gestützt einsetzen lassen, in denen die Web 2.0-Technologien im Sinne der Unterstützung der Kommunikation und des Austausches eine wichtige Rolle spielen können.

\section{Wie Wissensmanagement erfolgreich sein kann}

Sechs Faktoren sind es im wesentlichen, die für den Erfolg von Wissensmanagement entscheidend sind: rigen und 68 Prozent der 50- bis 64-Jährigen nutzen täglich beruflich oder privat das Internet (Durchschnitt über alle Altersgruppen ist 72 Prozent). Die 50bis 64-Jährigen nutzen das Internet $\mathrm{zu}$ etwa 50 Prozent ein bis zwei Stunden am Tag, während 47 Prozent der 14- bis 29-Jährigen zwei bis fünf Stunden pro Tag damit verbringen. 94 Prozent der 14- bis 29-Jährigen sind aktive Nutzer in einem sozialen Netzwerk, aber nur 47 Prozent der 50- bis 64-Jährigen. Etwa 25 Prozent der Nutzer verbringen dabei ein bis zwei Stunden am Tag darin. Der Anteil der mobilen Geräte für die Inter- netnutzung beträgt mittlerweile 18 bis 24 Prozent. Die Nutzung der Netzwerke erfolgt überwiegend privat. (Quelle: Repräsentative Bitkom-Studien: »Netzgesellschaft « und »Soziale Netzwerke«)

Doch wie sieht es in den Unternehmen aus? IT-Systeme, die die strukturierte Ablage von Informationen und den Austausch, die Kommunikation und Interaktion unterstützen, gewinnen auch hier an Bedeutung. Nicht zuletzt geht es um die Kommunikation mit den Kunden und sonstigen Partnern.

Wenn Wissen expliziert ist, werden Funktionen benötigt, mit deren Hilfe es strukturiert und systematisch abgelegt
Faktor Management: Austausch und Kommunikation müssen gelebt und gefördert werden. Das Management muss die Themen unterstützen.

- Faktor Unternehmenskultur als zentraler Faktor: Wissen muss geteilt werden wollen. Herrscht in der Organisation die Haltung, dass Wissen mit Macht zu tun hat, wird die Bereitschaft dieses teilen zu wollen, gering sein. Partizipation sollte als Prinzip gelebt werden und Vertrauen muss vorhanden sein. 
- Faktor Motivation und Anreizsysteme: Es muss sich für die Mitarbeitenden lohnen, sich zu beteiligen. Dabei ist nicht unbedingt an Geld zu denken. Anerkennung, Beteiligung, Verantwortung und Autonomie können ebenfalls motivierend wirken.

- Faktor Instrumente: Durch den Einsatz von geeigneten Instrumenten des Wissensmanagements können die Prozesse unterstützt werden. Die Instrumente müssen zur Unternehmenskultur passen.

- Faktor Informationstechnologie: Es bedarf geeigneter Software-Werkzeuge, die zu den Wissensprozessen passen. Ausstattung und Leistungsfähigkeit müssen angemessen sein.

- Faktor Regeln und Guidelines: Entscheidet man sich für Web 2.0-Instrumente, sollten Verhaltensregeln für Umgang und Nutzung definiert werden, nicht nur aus Gründen des Datenschutzes.

\section{Fazit}

Web 2.0 hilft bei Kommunikation und Interaktion. Grenzen bestehen jedoch in der Zusammenfassung und der Darstellung, die die individuelle Ebene verlässt und die Ergebnisse so standardisiert, dass sie nachvollziehbar werden.

Aus den genannten Zahlen lässt sich nicht schließen, dass Wissensmanagement mit Web 2.0-Technologien ohne weiteres auf den Einsatz in sozialen Organisationen übertragbar wäre. Ein Hinderungsgrund kann die Altersstruktur der Mitarbeitenden in sozialen Unternehmen sein. Dennoch wird deutlich, dass die Bekanntheit von Webanwendungen wie sozialen Netzwerken das Potenzial für Unternehmen steigert. Sie bietet Ansatzpunkte dafür, dass über die Vernetzung mittels IT-Systeme zweckund themenbezogen nachgedacht werden kann, zudem die Basiskenntnisse für die Nutzung von Webanwendungen mittlerweile breitflächig vorhanden sind.

Die jüngere Generation wird im Beruf nicht auf die Nutzung von Informationstechnologie in ihrer gesamten Breite verzichten wollen. Hier besteht für Unternehmen derzeit vielmehr das Risiko der Vermischung von Dienstlichem und Privatem, wenn zur Kommunikation interner Belange die privaten Kanäle genutzt werden.

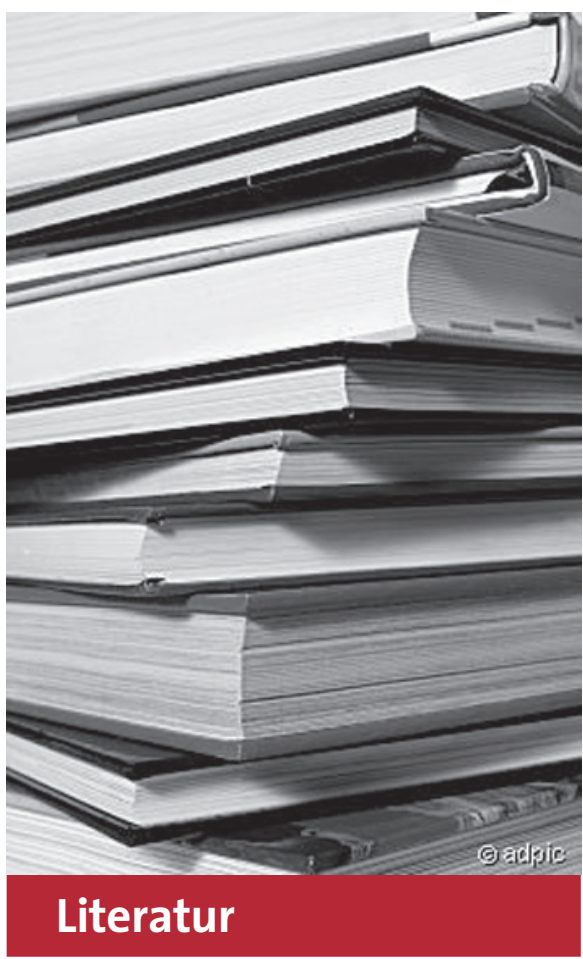

Hasler Roumois, Ursula 2007. Studienbuch Wissensmanagement: Grundlagen der Wissensarbeit in Wirtschafts-, Non-Profit- und PublicOrganisationen.

Kreidenweis, Helmut, Steincke, Willi: Wissensmanagement. Nomos Verlagsgesellschaft. Willke, Helmut 1998. Systemisches Wissensmanagement. Stuttgart: Lucius \& Lucius.

Nonaka, Ikujirō, Takeuchi, Hirotaka \& Mader, Friedrich 1997. Die Organisation des Wissens. Probst, Gilbert J., Raub, Steffen \& Romhardt, Kai 2006. Wissen managen: Wie Unternehmen ihre wertvollste Ressource optimal nutzen. 5. überarbeitete Auflage. Wiesbaden: Gabler. Bitkom: Internet http://www.bitkom.org/files/ documents/BITKOM_Publikation_Netzgesellschaft.pdf. Internet http://www.bitkom.org/ files/documents/BITKOM_Publikation_Soziale_Netzwerke.pdf.

\section{Aktuell: SGB II-Reform berücksichtigt.}
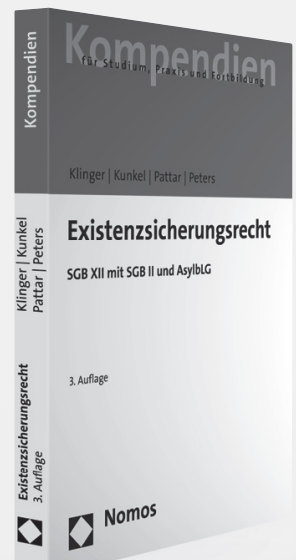

\section{Existenzsicherungsrecht}

SGB XII mit SGB II und AsylbLG

Von Prof. Roland Klinger, Prof. PeterChristian Kunkel, Prof. Dr. Andreas Kurt Pattar und Ri'in Karen Peters

3. Auflage 2012, 499 S., brosch., 28,- $€$ ISBN 978-3-8329-6265-4

Das Werk bietet eine systematische Darstellung des Existenzsicherungsrechts in SGB I, II, X, XII und des Asylbewerberleistungsgesetzes.

Außerdem enthält es die Hilfen in unterschiedlichen Lebenslagen nach dem SGB XII. Mit Prüfschemata und Schaubildern leistet es speziell Studierenden, aber auch Praktikern nützliche Arbeitshilfe.

www.nomos-shop.de/13263

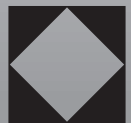

Nomos 